\title{
Follow the dummy: measuring the influence of a biomimetic robotic fish-lure on the collective decisions of a zebrafish shoal inside a circular corridor
}

\author{
Frank Bonnet, ${ }^{1}$ José Halloy, ${ }^{2}$ and Francesco Mondada ${ }^{1}$
}

\begin{abstract}
Robotic agents that are accepted by animals as conspecifics are very powerful tools in behavioral biology because of the ways they help in studying social interactions in gregarious animals. In recent years, we have developed a biomimetic robotic fish lure for the purpose of studying the behavior of the zebrafish Danio rerio. In this paper, we present a series of experiments that were designed to assess the impact of some features of the lure regarding its acceptance among the fish. We developed an experimental setup composed of a circular corridor and a motorized rotating system able to steer the lure inside the corridor with a tunable linear speed. We used the fish swimming direction and distance between the fish and the lure as a metric to characterize the level of acceptance of the lure, depending on various parameters. The methodology presented and the experimental results are promising for the field of animal-robot interaction studies.
\end{abstract}

\section{INTRODUCTION}

The mechanisms of interactions between individuals inside an animal society have always been one of the long-standing interests in behavioral research. It has been shown in [1] that animal communication can be based on rather simple signals and that it is possible to interact with animals by making specifically designed artifacts that generate and exploit only a part of the signals relevant for social behavior. At that time, the level of technology was not high enough to use more complex stimuli or multiple stimuli simultaneously. Rather simple dummies were used instead, each one serving to study only one specific behavior.

In recent years, technology has become more advanced and affordable, and such lures were replaced by approaches using robotized systems that are built and controlled according to the animal under study [2]. Among the social animals that are nowadays under these types of studies, small species of fish, such as zebrafish Danio rerio, guppies Poecilia reticulata, or stickleback Poecilia reticulata are often chosen as model for studying the collective behavior of fish and the types of interaction channels that they use in the collective decision-making process. Due to the fact that the communication channels used by fish during social interactions are mainly based on vision [3], screens are often used to evaluate the change of behavior of fish when changing the stimulus that is displayed, such as in [4][5]. However, these types of tests using screens are often limited

\footnotetext{
${ }^{1}$ F. Bonnet and F. Mondada are with the Robotic Systems Laboratory, School of Engineering, Ecole Polytechnique Fédérale de Lausanne, Lausanne, Switzerland frank. bonnet depfl.ch

${ }^{2}$ J. Halloy is with Université Paris Diderot VII, Sorbonne Paris Cité, LIED, UMR 8235, F-75205 Paris, France jhalloy@gmail.com
}

because they are not coupled with the motion induced in water, which fish are also very sensitive to [3]. Therefore, recent studies have used more complex systems that use robots to interact with fish by generating visual stimuli that are also moving underwater, thus inducing water waves. For instance, in [6], [7], [8], the response of zebrafish to a robotic fish was observed. The robotic fish, which had the same aspect ratio as the zebrafish, was attached to a moving device on top of a tank or moving autonomously, and its speed could be varied, along with the tail beating and its coloration. In [9], a study measured the preference of fertile female bluefin killifish Lucania goodei for a robotic replica whose aspect ratio, body size, motion pattern, and color were inspired by an adult male killifish; here, the authors used a robotic platform specifically designed to simulate the typical courtship behavior observed in male killifish. In [10], [11], [12], [13], [14], a passive lure attached to a support was moved using a mobile robot below the aquarium and controlled using tracking software. In [15], a mobile robot was used to move a robotic lure that emitted electrical playback signals to attract the weakly electric fish Mormyrus rume. In [16], the stickleback's schooling behavior was also observed using a carrousel that drove groups of stickleback lures. For several years, we have worked on developing robotic devices to study the collective behavior of zebrafish. We developed multi-robot systems to steer fish lures in an aquarium [17], [18], [19] and robotized fish lures to study the effect of the beating movements of the tail on the fish [20], [21].

In the current paper, we present a novel system to test the reaction of fish to different types of actuated and nonactuated lures. We propose the use of a circular corridor to measure the attraction of fish shoal to robotic lures that are equipped equipped with a soft beating tail. This setup offers a binary choice for the fish because they can either move in the clockwise $(\mathrm{CW})$ or counter-clockwise (CCW) direction. It is also a known setup to study the shoaling formation of zebrafish [22] or rummy-nose tetra Hemigrammus rhodostomus [23]. A coaxial motor is used to actuate two blades to move one or more lures inside the corridor, either $\mathrm{CW}$ or $\mathrm{CCW}$. The lure can have different ranges of linear speed, from 0 up to $40 \mathrm{cms}^{-1}$.

We measured the swimming direction of a shoal of five zebrafish when a lure that emitted different types of stimuli was moving inside the tank with the goal being to quantify the change in the collective decisions of the fish. The goal 
for the current study was to find the most attractive lure for further studies on the social integration of robotic fish into zebrafish societies.

The proposed experimental setup and the biological results obtained will help scientists to easily test the stimuli generated by robotic lures on fish.

\section{METhODS}

\section{A. Experimental Setup}

The experimental setup designed for the current study is presented in Fig. 1. For the experiment, a $1 \mathrm{~m} \mathrm{x} 1 \mathrm{~m}$ squared tank of $30 \mathrm{~cm}$ height was filled with water up to a level of $6 \mathrm{~cm}$. The tank was supported by four pillars of $1 \mathrm{~m}$ height. Underneath the tank, a coaxial motor moved two blades in the two possible directions, $\mathrm{CW}$ or $\mathrm{CCW}$. Magnets were placed at the tip of these blades to transmit the motion to the lures moving inside the tank. In the experiments, the lure was controlled in an open-loop; therefore, no multi-agent based tracking or complex controllers were required compared to other similar studies involving robotic fish.

To constrain the zebrafish, an arena composed of an outer and an inner circular wall was placed inside the tank, forming a circular corridor (Fig. 2). The dimensions of the corridor were as follows: an external diameter of $58 \mathrm{~cm}$, an internal diameter of $38 \mathrm{~cm}$, and thus a corridor width of $10 \mathrm{~cm}$. The choice of the $10 \mathrm{~cm}$ width was a good trade-off, allowing the zebrafish to have continuous motion without being stressed. Indeed, in a large area, the zebrafish would tend to either move along the walls or stay in one place due to stress, but in a very narrow corridor, they continuously move in one of the two possible directions.

(a)

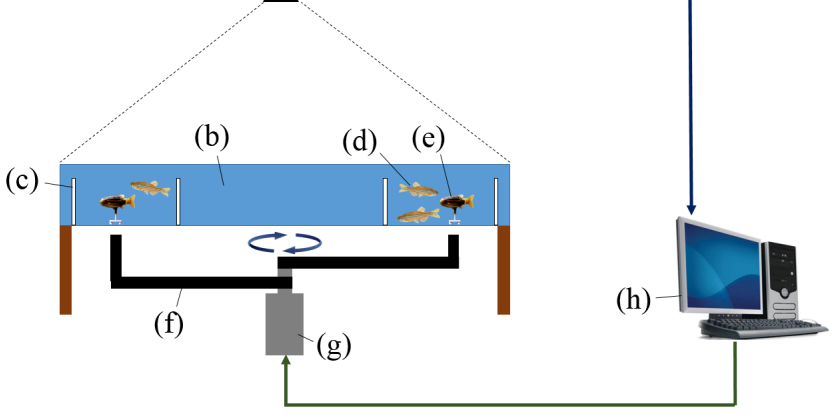

Fig. 1. Experimental setup used for the current study. A coaxial motor rotated two blades underneath a squared tank of $1 \mathrm{~m} \times 1 \mathrm{~m} \times 0.3 \mathrm{~m}$. The two blades could rotate in both directions independently and in a different range of speeds that could be controlled from a computer. Inside the tank, the fish and the lures were constrained in a circular corridor. A camera was placed on top of the tank and grabbed video frames that were collected on a computer. a) Camera. b) Tank filled with water. c) Wall of the circular arena. d) Zebrafish. e) Biomimetic robotic fish-lure RiBot. f) Blade. g) Motor. h) Computer.

\section{B. Zebrafish}

The experiments performed in the current study were conducted under authorization N. 2778 delivered by the Department of Consumer and Veterinary of the Canton de Vaud

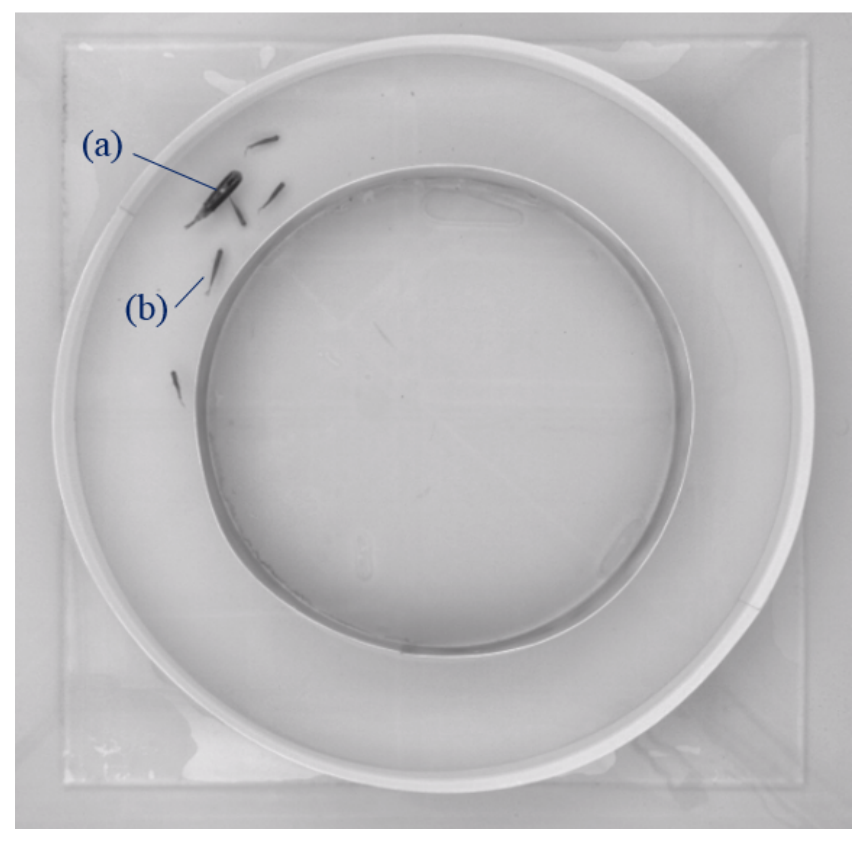

Fig. 2. Top view of the experimental setup, with the lure moving among the group of five zebrafish inside the circular corridor. a) Biomimetic robotic fish lure RiBot. b) Zebrafish.

(Switzerland) after submission to the state ethical board for animal experiments. For the experiments performed, we used 100 wild-type zebrafish Danio rerio, with short fins. These zebrafish were acquired in a pet shop, and were stored in a 60 -liter housing aquarium. The average total length of our zebrafish was $40 \mathrm{~mm}$. The water temperature of the housing aquarium was $26^{\circ} \mathrm{C}$. The fish were fed twice a day with commercial food using a food distributor. The enrichment in the aquarium consisted of plastic plants, cladophoras, gravel, rocks, and aquatic snails.

The fish were randomly selected from their housing aquarium and then transferred to the experimental tank. The zebrafish were used for only one experiment per day. It is possible that some fish were tested several times for the same conditions.

We used preliminary experiments to determine the optimal number of fish required. Table I shows a comparative study of the different shoal sizes that were tested in the preliminary experiments. With a shoal size smaller than five individuals, the group was very homogenous, but, due to the low number of fish, there was a lower chance that one of them would be attracted by the robot and hence a lower chance to see an effect on the whole group. There was also a high chance that the fish would freeze, probably because they usually live with many other fish in their housing aquarium and felt stressed when there was only a few of them in an open area. For the case of shoal with more than five fish, we observed that the effect of the robot on individuals was increased; however, the homogeneity of the group decreased as the number of fish increased. We observed that the best trade-off between the observed effect and homogeneity was for groups of five 
fish; therefore, we performed the experiments presented in this study with only groups of five zebrafish.

\section{Lures}

The RiBot biomimetic fish lure design had already been described in detail in [20] and [21] and is presented in Fig. 3. This device was designed based on the 3D scan of a zebrafish and mimics the external shape of the animal. The RiBot is 1.5 times longer than the average length of our zebrafish. It is equipped with a stepper motor to reproduce the tail beating movements of zebrafish. The tail of the RiBot is made of soft latex that allows it to move, and the upper part of the device is molded inside polyurethane.

The RiBot is not able to swim autonomously underwater, so it is linked via magnetic coupling to the blade driven by the motor underneath the tank. For the control of the RiBot's tail beating movements, a remote control that generated infrared RC5 signals was broadcast over the whole aquarium to control the RiBots that are equipped with an infrared receiver. The tail beating movements were programmed to change according to a given message.

The RiBot was moving at a constant water level that depended on the height of the robot's support. We chose a support height of $3 \mathrm{~cm}$ to have the lure moving in the middle of the water level. The lures were placed on a module composed of a carbon pin that was attached to an iron plate on which two magnets were placed. The module was painted in white so that it blended in with the white background of the tank. For the colored lure, the zebrafish pattern was printed on the skin of the lure using decals. The pattern was then covered with latex to make it waterproof.

The linear speed of the lure for each experiment was 12 $\mathrm{cms}^{-1}$. This speed was higher than the average speed of the fish in the circular corridor $\left(8 \mathrm{cms}^{-1}\right)$ because we wanted to increase the attractive effect of the lure. Indeed, when escaping from the group and possibly attracting the rest of the shoal with them, the zebrafish has a tendency to increase its linear speed [24], and thus, a robot with a linear speed higher than the zebrafish shoal could have a higher chance of attracting the zebrafish.

In the current study, we tested two aspects of the lure on the reaction of the fish: the actuation of the tail and the

TABLE I

QUALITATIVE EFFECT OF THE SHOAL SIZE ON THE DIFFERENT PARAMETERS INVOLVED IN THE MEASUREMENTS OF THE COLLECTIVE BEHAVIOR OF THE ZEBRAFISH IN EXPERIMENTS INVOLVING A CIRCULAR CORRIDOR (++: OPTIMAL RESULT, +: GOOD RESULT, -: POOR RESULT, - -: VERY POOR RESULT).

\begin{tabular}{|l|c|c|c|}
\hline $\begin{array}{l}\text { Number } \\
\text { of fish }\end{array}$ & $\begin{array}{c}\text { Group's } \\
\text { homogeneity }\end{array}$ & $\begin{array}{c}\text { Attraction } \\
\text { effect }\end{array}$ & $\begin{array}{c}\text { Risk } \\
\text { of freezing }\end{array}$ \\
\hline 1 & ++ & -- & -- \\
2 & ++ & - & - \\
5 & + & + & ++ \\
8 & - & ++ & ++ \\
10 & -- & ++ & ++ \\
\hline
\end{tabular}

coloration. It is known that both of these aspects can have an impact on the reactions of the zebrafish [6]; therefore, we wanted to assess which of the two parameters could have the most impact on attraction using our robotic lure RiBot, which dimensions is more close to the one in [6].

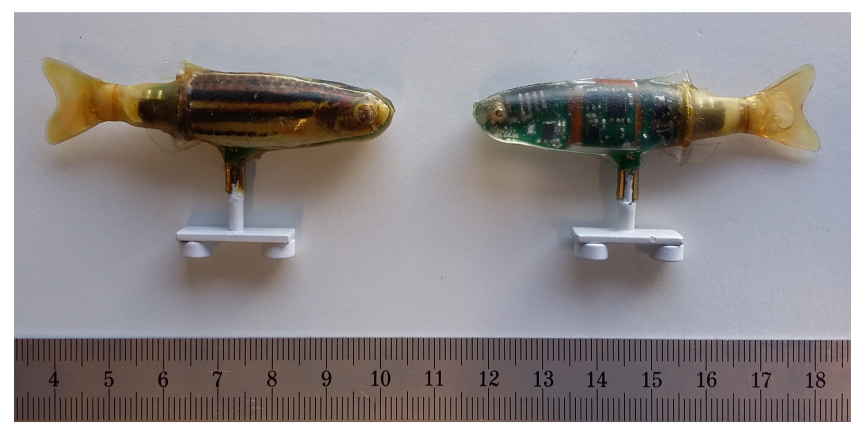

Fig. 3. Actuated biomimetic lure RiBot used in the experiments. Left: Lure with a zebrafish color pattern. The pattern was printed on the skin of the lure using decals. The pattern was then covered with latex to become waterproof. Right: Lure without any zebrafish color pattern. The PCB with all the electronic components that are used for the actuation of the caudal peduncle can be seen from the outside.

\section{Tracking and data analysis}

We used an overhead acA2040-25gm monochrome GigE CCD camera (Basler AG, Germany) with a maximum resolution of $2048 \mathrm{px} \times 2048 \mathrm{px}$ and equipped it with low distortion lenses CF12.5HA-1 (Fujinon, Tokyo, Japan) to grab high resolution frames that were later processed on a computer. The high-resolution videos were then processed with the open-source software idTracker [25]. This allowed us to track and identify the zebrafish. This process is timeconsuming and computationally intensive (idTracker tracks and identifies five fish in $30 \mathrm{~min}$ high definition videos in 1 day) but relatively reliable: there are no false positives and no propagation of identification errors, and the fish are identified correctly in $95 \%$ of time-steps on average.

The identification of the fish allowed for the measurement of the individual swimming direction and the distance between the individual fish and the lure, which was used to measure the attractiveness of the robotic fish lure to the fish shoal.

\section{E. Experiments' description}

We assessed the effect of the actuated tail and color appearance of the RiBot lure on the collective decision of the zebrafish. The tail was actuated with a frequency of 5 $\mathrm{Hz}$ and an amplitude of \pm 5 degrees. The choice of the tail beating frequency and amplitude was made based on preliminary experiments that were presented in [20] tested the attraction of the lure for fish to different ranges of frequencies and amplitudes. The two RiBots that were used during this experiment can be seen in Fig. 3.

The RiBot can beat its tail thanks to a stepper motor, which also generates some noise when activated. First, we tested whether the noise of the motor had an effect on the collective 
decision of the zebrafish. We decoupled the caudal peduncle part from the motor to activate the motor without generating any movements of the tail. We thus tested the first three conditions to determine whether the lure and motor sound could influence the collective movements of the fish.

- no lure in the setup (No Lure)

- lure without a pattern and motor not moving (Motor OFF)

- lure without a pattern and motor moving without the tail fixed to the motor (Motor ON)

Then, on a second series of experiments, we tested four conditions to determine the attractive factors of the RiBot among the visual appearance and the tail beating:

- lure with pattern and tail not moving (Tail ON + Pattern)

- lure with pattern and tail moving (Tail ON)

- lure without pattern and tail not moving (Tail OFF + Pattern)

- lure without pattern and tail moving (Tail OFF)

\section{F. Results and discussion}

Figure 4 shows a comparison between an experiment in which no lure was moving in the setup, and experiments with the lure, one in which the stepper motor was not activated and one with the motor activated without the tail beating movements. First, inside a circular corridor, without any object rotating, and during the 30-minutes experiments, a shoal composed of five zebrafish had a tendency to swim half of the time $\mathrm{CW}$ and half of the time CCW. We also found a significant difference for the distribution between the three focal groups (Kruskal-Wallis, $\mathrm{p}<0.05$ ). A post hoc analysis demonstrated that the mean ranks of the first condition were significantly different from the two other conditions because the two conditions involving the lure had no significantly different distributions. It thus shows that a lure moving in this setup is able to influence the swimming direction of a group of fish and that the motor-generated vibrations had no significant effect on the swimming direction of the zebrafish.

Figure 5 shows the result of the tests for the four conditions using the two types of RiBots and the actuation of the tail: RiBot with a pattern and tail on, RiBot without a pattern and tail on, RiBot with pattern and tail off, and RiBot without a pattern and tail off.

We computed the relative effect of each factor: the effect of the motor $a_{1}$, the pattern $a_{2}$, and the combination of both effect $a_{12}$. Table II shows the relative effects of the factors. Both $a_{1}$ and $a_{12}$ have a relative effect close to 5\%, compared with $a_{2}$, which seems to have a lower effect on the swimming direction of the zebrafish. This shows that the movements of the tail seems to be a more important factor than the visual appearance of the lure. However, the latter also seems to play a role in the acceptance of the device. We performed an ANOVA on the data to assess whether one of the parameters could have a significant impact on the change in the collective decisions of the fish. The results of this ANOVA can be seen in Table III. The only factor

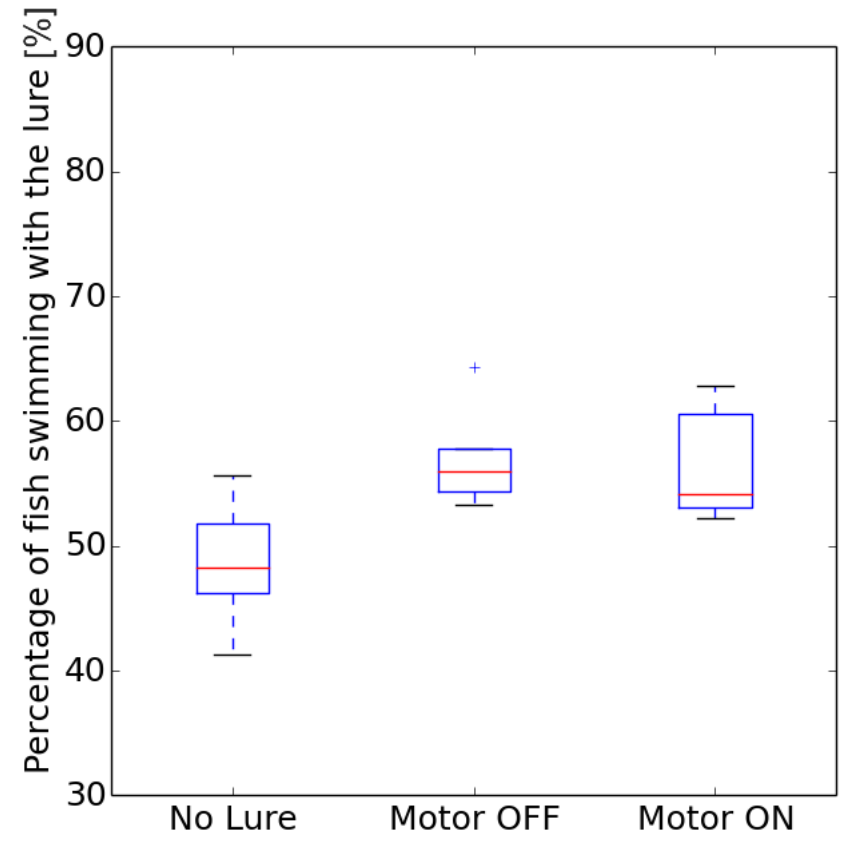

Fig. 4. Effect of the noise of the motor on the swimming preference of the zebrafish. Each condition was tested six times with a trial duration of 30 minutes each. The fish were randomly selected from their housing aquarium. The zebrafish were used for only one experiment. The distributions differ significantly (Kruskal-Wallis $\mathrm{p}<0.05$ ).

that had a significant impact is the beating tail $a_{1}(\mathrm{p}<0.05)$, confirming that this factor plays a non-negligible role in the attraction of the zebrafish.

TABLE II

RELATIVE EFFECT OF THE DIFFERENT FACTORS ON THE SWIMMING DIRECTION OF THE ZEBRAFISH.

\begin{tabular}{|l|c|c|}
\hline Factor & ID & Relative effect (\%) \\
\hline Tail beating & $a_{1}$ & 4.54 \\
Pattern & $a_{2}$ & 0.74 \\
Tail beating and pattern & $a_{12}$ & 2.97 \\
\hline
\end{tabular}

Figures 6 and 7 show the percentage of swimming direction for individual fish versus the median distance between individual fish and the RiBot for the experiments involving five zebrafish and the RiBot that has the tail ON (Fig. 6) and the tail OFF (Fig. 7). For the experiment involving the lure beating its tail, there is a correlation between the individual fish lure distance and the swimming direction of the fish. However, this does not seem to be the case for experiments with the RiBot not moving its tail. A linear fitting regression was applied to the two different conditions, showing that there is a significant correlation between the lure-fish distance and the swimming direction of the fish for the experiments with the tail of the RiBot ON $(p<0.05)$, which is not the case for the experiments with the tail OFF ( $p>0.05)$. Thus, for a lure able to change the collective decisions of the fish, the fish that are close to the lure seem also to be the ones that are mostly swimming with it, which demonstrates the attractiveness of the device. 


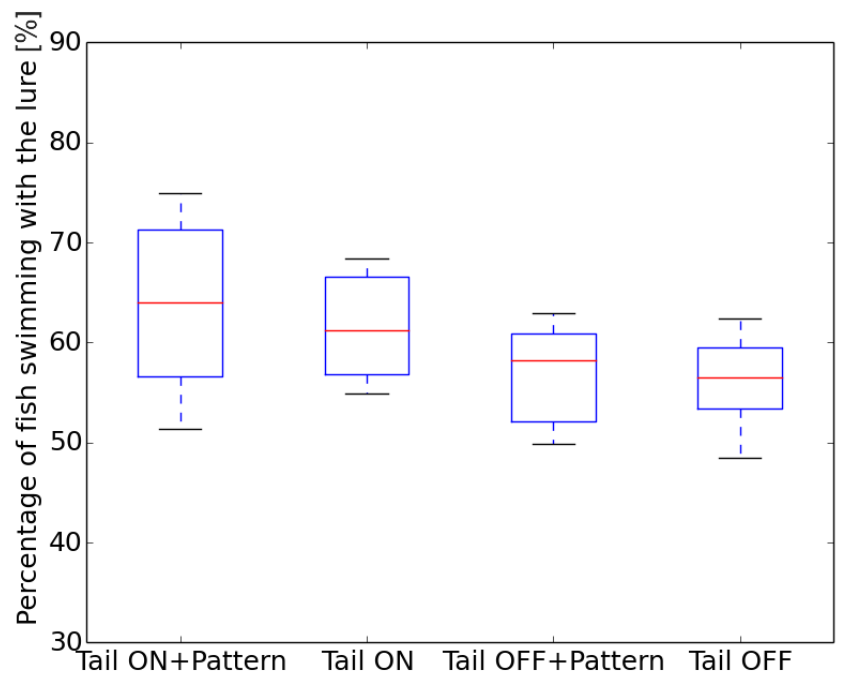

Fig. 5. Swimming direction preference of the fish with the four conditions tested: the lure with a pattern beating the tail (Tail ON+Pattern), the lure without a pattern beating the tail (Tail ON), the lure with a pattern not beating the tail (Tail OFF+Pattern), and the lure without a pattern not beating the tail (Tail OFF). Each condition was tested six times. The fish were randomly selected from their housing aquarium. The fish were used for only one experiment per day.

TABLE III

ANOVA OBTAINED FOR EXPERIMENT 4. $a_{1}$ CORRESPONDS TO THE BEATING TAIL FACTOR, $a_{2}$ TO THE COLOR PATTERN FACTOR, AND $a_{12}$ TO THE INTERACTION TERM.

\begin{tabular}{lrcrrr}
\hline Effect & SS & df & MS & F & p \\
\hline$a_{1}$ & 265.07 & 1 & 265.069 & 6.79 & 0.0169 \\
$a_{2}$ & 4.05 & 1 & 4.051 & 0.1 & 0.7506 \\
$a_{12}$ & 16.1 & 1 & 16.105 & 0.41 & 0.5279 \\
Residual & 780.29 & 20 & 39.015 & & \\
\hline Total & 1065.52 & 23 & & & \\
\hline
\end{tabular}

\section{CONCLUSION AND FUTURE WORKS}

In this study, we proposed the design of an experimental setup to test the acceptance of lures by small species of fish. Small groups of zebrafish were constrained inside a circular corridor and moved either $\mathrm{CW}$ or $\mathrm{CCW}$. Because the fish were constantly swimming, this measure appeared to be reliable for identifying the change of collective choices relative to the motion of the robotic lure.

We showed that having a lure inside a corridor and moving at a speed above the average linear speed of the fish could trigger an effect on the zebrafish shoal's direction of swimming. The five fish shoal's size was a good trade-off between homogeneity and the effect that was observed. We studied the effect of an actuated biomimetic lure by using the beating of the tail and the coloration as parameters to study the attractiveness of the device. We showed that the tail beating had a significant effect on the attraction of the fish towards the lure when compared with the coloration.

We also showed that there was a correlation between the individual fish lure distance and the swimming direction of the fish when the fish were swimming more in the direction

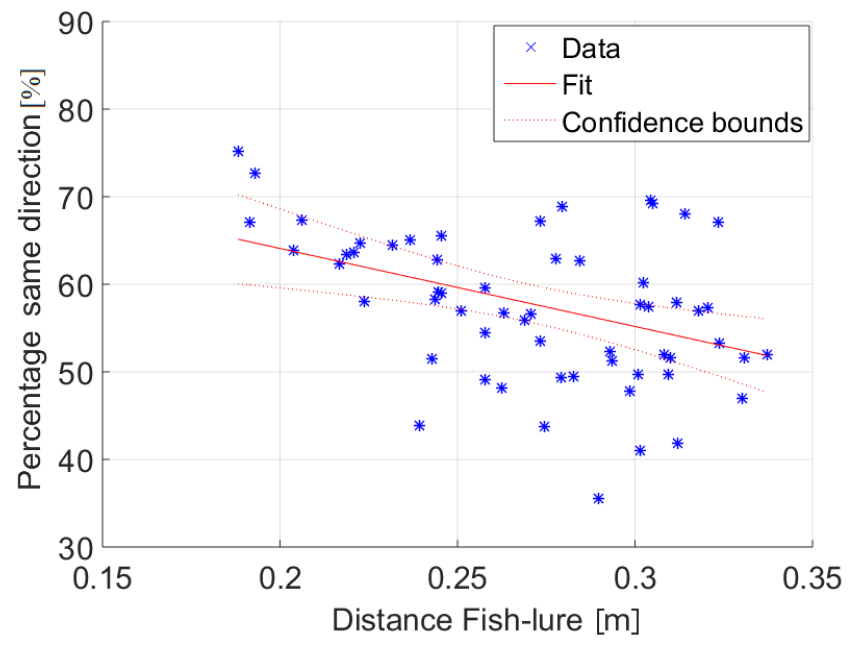

Fig. 6. Linear regression for the individual fish-RiBot distance versus the fish swimming direction for RiBot ON for the experiments involving five zebrafish and the actuated lure moving in the circular corridor with the tail beating $(\mathrm{p}<0.05)$. The red line indicates the linear regression that was performed on the data.

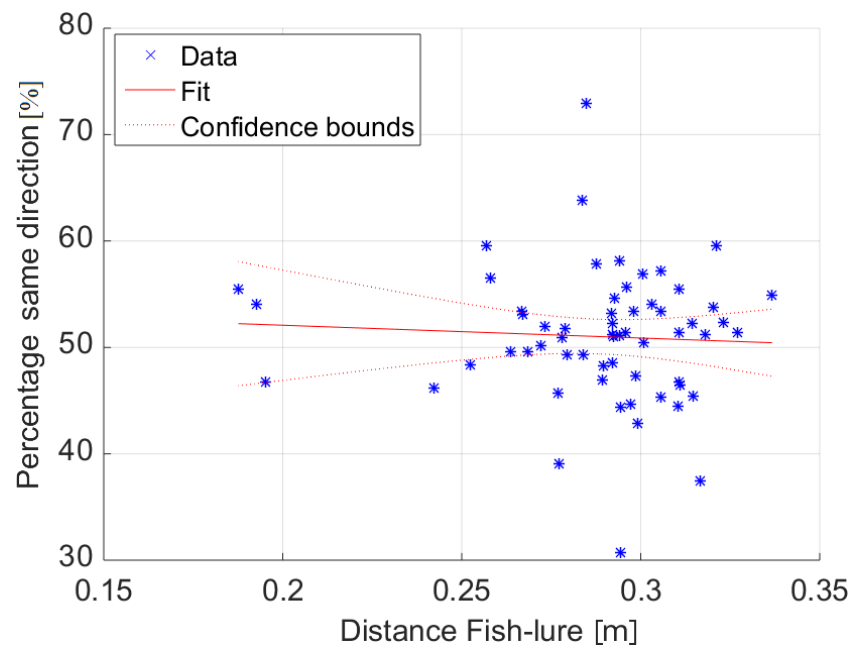

Fig. 7. Linear regression for the individual fish-RiBot distance versus the fish swimming direction for RiBot OFF for the experiments involving five zebrafish and the actuated lure moving in the circular corridor with the tail not beating $(\mathrm{p}>0.05)$. The red line indicates the linear regression that was performed on the data.

of the lure. This shows that the closer the fish are to the lure, the more they are swimming in the same direction. Hence, the decision of the zebrafish is not influenced by the fact that they are frightened by the lure; it is the lure itself that influences the fishes' decision. This measure could also be a good indication of the acceptance of a lure among a group of fish in this type of experimental setup.

We were able to show that to modify the collective decision significantly to target mixed society experiments, the use of lures that are biomimetic and equipped with a beating tail would increase the attractiveness of the device. This was already shown, for instance in [6]; however, here, we were able to show it with a smaller robotized fish lure 
that was only 1.5 times the size of a zebrafish. All these results confirmed that a lure designed to perform experiments involving mixed societies of fish and robots should include two main features to increase the attraction of the lure: a biomimetic shape and a beating tail, which shows the potential of designing miniature and sophisticated robots based on soft-robotics techniques that can mimic the animal's behavior.

In further work, we plan to use the two blades to test the effect of multiple lures moving in two directions inside the circular corridor on the collective decisions of the fish. We plan also to use the position feedback of the motors to control the lures in a closed loop according to the behavior of the fish, such as the swimming direction, the speed or the group cohesion. Finally, we will perform a deeper analysis on the interaction between the agents, for instance by using information theory, to gain new knowledge on the decisionmaking process in fish shoals.

\section{ACKNOWLEDGEMENT}

This work was supported by the EU-ICT project ASSISIbf, No. 601074. The information provided is the sole responsibility of the authors and does not reflect the European Commission's opinion. The European Commission is not responsible for any use that might be made of the data appearing in this publication. We would like to gratefully acknowledge Daniel Burnier and Norbert Crot for their technical support during the design and production of the actuated lure.

\section{REFERENCES}

[1] N. Tinbergen, The Study of Instinct. Oxford University Press, Oxford, 1951.

[2] J. Krause, A. F. Winfield, and J.-L. Deneubourg, "Interactive robots in experimental biology," Trends in Ecology and Evolution, vol. 26, no. 7, pp. 369 - 375, 2011. [Online]. Available: http://www.sciencedirect.com/science/article/pii/S0169534711000851

[3] T. J. Pitcher and J. K. Parrish, "Functions of shoaling behaviour in teleosts," in The behaviour of teleost fishes. Chapman and Hall, London, 1993, pp. 294-337.

[4] M. Qin, A. Wong, D. Seguin, and R. Gerlai, "Induction of social behavior in zebrafish: live versus computer animated fish as stimuli," Zebrafish, vol. 11, no. 3, pp. 185-197, 2014.

[5] S. L. Bass and R. Gerlai, "Zebrafish (danio rerio) responds differentially to stimulus fish: the effects of sympatric and allopatric predators and harmless fish," Behavioural Brain Research, vol. 186, no. 1, pp. 107-117, 2008.

[6] N. Abaid, T. Bartolini, S. Macri, and M. Porfiri, "Zebrafish responds differentially to a robotic fish of varying aspect ratio, tail beat frequency, noise, and color," Behavioural brain research, vol. 233, no. 2 , pp. 545-553, 2012.

[7] M. Aureli, F. Fiorilli, and M. Porfiri, "Portraits of self-organization in fish schools interacting with robots," Physica D: Nonlinear Phenomena, vol. 241, no. 9, pp. 908 - 920, 2012. [Online]. Available: http://www.sciencedirect.com/science/article/pii/S016727891200036X

[8] S. Butail, G. Polverino, P. Phamduy, F. Del Sette, and M. Porfiri, "Fishrobot interactions in a free-swimming environment: Effects of speed and configuration of robots on live fish," vol. 9055, 2014, pp. 90 550I90 550I-7. [Online]. Available: http://dx.doi.org/10.1117/12.2044622

[9] P. Phamduy, G. Polverino, R. Fuller, and M. Porfiri, "Fish and robot dancing together: bluefin killifish females respond differently to the courtship of a robot with varying color morphs," Bioinspiration \& biomimetics, vol. 9, no. 3, p. 036021, 2014.
[10] S. Marras and M. Porfiri, "Fish and robots swimming together: attraction towards the robot demands biomimetic locomotion," Journal of The Royal Society Interface, vol. 9, no. 73, pp. 1856-1868, 2012. [Online]. Available: http://rsif.royalsocietypublishing.org/content/9/73/1856

[11] J. J. Faria, J. R. Dyer, R. O. Clément, I. D. Couzin, N. Holt, A. J. Ward, D. Waters, and J. Krause, "A novel method for investigating the collective behaviour of fish: introducing robofish," Behavioral Ecology and Sociobiology, vol. 64, no. 8, pp. 1211-1218, 2010.

[12] T. Landgraf, H. Nguyen, S. Forgo, J. Schneider, J. Schröer, C. Krüger, H. Matzke, R. O. Clément, J. Krause, and R. Rojas, Advances in Swarm Intelligence: 4th International Conference, ICSI 2013, Harbin, China, June 12-15, 2013, Proceedings, Part I. Berlin, Heidelberg: Springer Berlin Heidelberg, 2013, ch. Interactive Robotic Fish for the Analysis of Swarm Behavior.

[13] T. Landgraf, D. Bierbach, H. Nguyen, N. Muggelberg, P. Romanczuk, and J. Krause, "Robofish: increased acceptance of interactive robotic fish with realistic eyes and natural motion patterns by live trinidadian guppies," Bioinspiration \& biomimetics, vol. 11, no. 1, p. 015001, 2016.

[14] D. T. Swain, I. D. Couzin, and N. E. Leonard, "Real-time feedbackcontrolled robotic fish for behavioral experiments with fish schools," Proceedings of the IEEE, vol. 100, no. 1, pp. 150-163, Jan 2012.

[15] M. Worm, T. Landgraf, H. Nguyen, and G. von der Emde, "Electrocommunicating dummy fish initiate group behavior in the weakly electric fish mormyrus rume," in Conference on Biomimetic and Biohybrid Systems. Springer, 2014, pp. 446-448.

[16] A. R. Wark, A. K. Greenwood, E. M. Taylor, K. Yoshida, and C. L. Peichel, "Heritable differences in schooling behavior among threespine stickleback populations revealed by a novel assay," PloS one, vol. 6, no. 3, p. e18316, 2011.

[17] F. Bonnet, P. Retornaz, J. Halloy, A. Gribovskiy, and F. Mondada, "Development of a mobile robot to study the collective behavior of zebrafish," in 2012 4th IEEE RAS EMBS International Conference on Biomedical Robotics and Biomechatronics (BioRob), June 2012, pp. $437-442$.

[18] F. Bonnet, S. Binder, M. E. de Oliveria, J. Halloy, and F. Mondada, "A miniature mobile robot developed to be socially integrated with species of small fish," in Robotics and Biomimetics (ROBIO), 2014 IEEE International Conference on, Dec 2014, pp. 747-752.

[19] F. Bonnet, A. Gribovskiy, J. Halloy, and F. Mondada, "Closed-loop interactions between a shoal of zebrafish and a group of robotic fish in a circular corridor," Swarm Intelligence, pp. 1-18, 2018.

[20] F. Bonnet, Y. Kato, J. Halloy, and F. Mondada, "Infiltrating the zebrafish swarm: design, implementation and experimental tests of a miniature robotic fish lure for fish-robot interaction studies," Artificial Life and Robotics, vol. 21, no. 3, pp. 239-246, 2016. [Online]. Available: http://dx.doi.org/10.1007/s10015-016-0291-8

[21] F. Bonnet, N. Crot, D. Burnier, and F. Mondada, "Design methods for miniature underwater soft robots," in 2016 6th IEEE International Conference on Biomedical Robotics and Biomechatronics (BioRob), June 2016, pp. 1365-1370.

[22] N. Abaid and M. Porfiri, "Fish in a ring: spatio-temporal pattern formation in one-dimensional animal groups," Journal of The Royal Society Interface, p. rsif20100175, 2010.

[23] V. Lecheval, L. Jiang, P. Tichit, C. Sire, C. K. Hemelrijk, and G. Theraulaz, "Domino-like propagation of collective uturns in fish schools," bioRxiv, 2017. [Online]. Available: http://biorxiv.org/content/early/2017/05/19/138628

[24] B. Collignon, A. Séguret, Y. Chemtob, L. Cazenille, and J. Halloy, "Collective departures in zebrafish: profiling the initiators," arXiv preprint arXiv:1701.03611, 2017.

[25] A. Pérez-Escudero, J. Vicente-Page, R. C. Hinz, S. Arganda, and G. G. de Polavieja, "idtracker: tracking individuals in a group by automatic identification of unmarked animals," Nature methods, vol. 11, no. 7, pp. 743-748, 2014. 